\title{
Dispersión y suburbanización. Nuevos paisajes étnicos
}

\author{
Pau Serra del Pozo \\ Universidad Internacional de Catalunya \\ pserra@cir.uic.es
}

Resum: Las divergencias respecto al sencillo esquema de los enclaves étnicos o centralidades étnicas son numerosas en la actualidad, incluso en la Little Havana de Miami. Tres procesos tienden a diluir ese esquema: a) la descentralización hacia los suburbios o la periferia urbana de algunos residentes inmigrantes, en ocasiones más consolidados o acomodados que los inmigrantes más recientes, que tienden a alojarse en el centro urbano; $b$ ) la dispersión de emprendedores de una determinada minoría étnica en una extensa área metropolitana; c) la aparición y convivencia de múltiples grupos étnicos en una misma zona o barrio. Los tres procesos provocan la pérdida progresiva de la unidad del tándem coétnico residentes-emprendedores en un mismo barrio.

Palabras clave: descentralización, suburbanización, emprendedores inmigrantes.

Abstract: There are many deviations from the simple scheme of ethnic enclaves or ethnic centralities today, even in contemporary Little Havana in Miami. This scheme tends to be diluted by three processes: 1) the decentralization of some immigrant residents towards the suburbs or urban periphery; these suburban immigrants may be more settled or affluent than recent immigrants living in inner-city communities; 2) the dispersal of entrepreneurs of a particular ethnic minority in a large metropolitan area; and 3) the emergence and coexistence of multiple ethnic groups in the same zone or area. The three processes gradually cause the loss of the unity of the residententrepreneur coethnic tandem in the same neighbourbood.

Keywords: decentralization, suburbanization, immigrant entrepreneurs 


\section{Introducción}

¿Existen excepciones a la regla de la «centralidad étnica» ${ }^{1}$ - residentes inmigrantes o de un determinado grupo étnico que se concentran en un barrio donde también se localizan emprendedores del mismo grupo-? A continuación intentaré presentar las tipologías de espacios de negocios étnicos diferentes de las «centralidades étnicas». Estos espacios son: centralidades étnicas minoritarias, centralidades multiétnicas minoritarias y centralidades étnicas especializadas.

La disociación espacial entre las zonas residenciales de inmigrantes y las concentraciones de negocios coétnicos y los espacios de socialización coétnica responde al fenómeno del heterolocalism, un modelo de asimilación social y espacial alternativo al asimilacionista y al multicultural (Zelinsky y Lee, 1998; Li, 2007). Para Raulin (2000), esta separación no es ajena a la sociedad mayoritaria, sino que manifiesta los procesos crecientes desde el siglo XIX hacia la separación de zonas funcionales - para trabajar, para residir, para comprar, para divertirse, etc.-, como lo muestra el caso de la Petite Asie, donde se concentran numerosos supermercados y pequeños comercios asiáticos ( $y$, en cambio, escasos residentes asiáticos) en un barrio periférico de París renovado, bien comunicado por transporte público y por grandes avenidas, cercano al périphérique, o cinturón de ronda que envuelve París y lo hace accesible a los asiáticos parisinos y los procedentes de la banlieue.

Rekers y Van Kempen (2000: 62) señalan la posibilidad de la separación entre las zonas de residencia de los inmigrantes y las zonas donde los emprendedores coétnicos tienden a concentrarse:

It is also possible for a cluster of ethnic entrepreneurs to stay put while the original population moves on, as happened in the Chinatowns of New York and Los Angeles.

En cuanto a las excepciones a las centralidades étnicas hay que decir, en primer lugar, que en las «centralidades étnicas minoritarias» no se produce una correspondencia entre la nacionalidad o el origen étnico del grupo de negociantes predominante y el origen étnico de la mayoría o de los diversos grupos de residentes de la zona. Es decir, las «centralidades étnicas minoritarias» constituyen concentraciones de negocios de titulares de un grupo étnico en un entorno residencial no dominado por los compatriotas de los negociantes.

1 En mi anterior artículo en RIO traté de describir las «centralidades étnicas» («Los procesos geográficos de concentración en las centralidades étnicas». RIO, junio de 2009: 89-110). 
En Sydney (Collins, 2006), Chinatown presenta una alta concentración de residentes de origen chino y abundantes negocios coétnicos. Sin embargo, otras dos activas centralidades étnicas de esa ciudad, como son Little Italy y Asiatown, no cuentan, en cambio, con una mayoría de población coétnica residente. Dentro del área metropolitana de Sydney, en el municipio de Leichhardt — que alberga Little Italy- concretamente, en 1961 vivían 6.000 italianos; en 1981 solo tenía 1.000. Para Collins está claro que no son los residentes italianos los que mantienen activa ahora y antes la centralidad, sino los emprendedores de esa nacionalidad:

It is not the population of Leichhardt that confers on it the status of an ethnic precinct but rather the first and second-generation Italian immigrant entrepreneurs who dominate the retail shopping and restaurant businesses in Leichhardt municipality than Italian-born immigrants. (Collins, 2006: 141).

En el Koreatown de la ciudad de Los Ángeles, dos tercios de los habitantes son «latinos» y solo una quinta parte coreanos. Lo que caracteriza a esa Koreatown no es lógicamente la etnicidad de la mayoría de sus residentes sino la concentración de las actividades económicas de coreanos, sean quienes sean los clientes. De hecho, los bancos coreanos en Koreatown no solo atienden a la población coreana sino también a la hispana (Li et alii, 2006). Además, la «escasez» de población coreana en Koreatown queda compensada por el atractivo que ejerce entre los coreanos de otros barrios de Los Ángeles, que acuden ahí para comprar, comer y entretenerse (Light y Bonacich, 1988, citados por Waldinger et alii, 1990; Zhou, 2004).

Eden Center, en los suburbios de Washington D. C. (en Virginia del Norte), es un shopping mall vietnamita con 120 grandes almacenes. El 95\% de los cerca de 40.000 vietnamitas que residen en el área metropolitana de Washington D.C. residen de manera heterolocal en cuatro condados suburbanos, donde no constituyen en ningún caso más del $9 \%$ en cada distrito censal (Airriess, 2007).

En ocasiones, las «centralidades étnicas minoritarias» pueden consistir en concentraciones de emprendedores étnicos que han permanecido en su antiguo enclave residencial tras la salida de sus coétnicos. Los antiguos enclaves judíos en el Lower East Side de Manhattan, en el Marais o Pletzl de París y en el East End de Londres constituían todavía en los años ochenta del pasado siglo importantes centros culturales y comerciales judíos (Waldinger et alii, 1990). Efectivamente, los judíos residentes en el Quartier Juif de París, en la rue Rosiers y alrededores, no constituyen más que el 25\% del total de los habitantes de la zona (Brody, citado por Raulin, 2000), mientras que los comercios judíos constituyen la mayoría de los negocios en el Quartier, junto a otros espacios étnicos como las sinagogas. 
Para Djergaïan (2007), el barrio del faubourg de Montmartre de París, animado entre 1920 y 1970 aproximadamente por los inmigrantes armenios que huían de la persecución en su país, constituye todavía hoy en día un «territorio identitario». Aunque la mayoría de los residentes armenios ya han abandonado el barrio, aún mantienen ahí sus joyerías y sus asociaciones.

Waldinger et alii (1990) señalan efectivamente el carácter evolutivo y cambiante de los negocios y los barrios étnicos: lo que antes podía ser una «centralidad étnica» puede pasar a convertirse en una «centralidad étnica minoritaria»: en antiguos barrios de judíos como el South Side de Chicago, o Harlem en New York, o en el East Side de Detroit (reocupados por negros inmigrantes), los comerciantes judíos pasaron a tener como clientes a población afroamericana. De manera similar, cuando los enclaves cubanos de los años sesenta en Nueva York perdieron su población, que fue sustituida por dominicanos y otros latinoamericanos, los negocios cubanos permanecieron. Pero los mismos autores incluyen no solo a coreanos sino también a japoneses (antes de la II Guerra Mundial), indios, árabes y chinos, que se han adaptado a barrios de residentes negros ${ }^{2}$ o hispanos en las ciudades de los Estados Unidos.

Los emprendedores inmigrantes caribeños de las Indias Occidentales han tendido a instalarse en barrios de negros en las ciudades de EE.UU., donde buscan una demanda que, sin ser absolutamente coétnica, iba a aceptarles mejor por el color de su piel (Boswell y Jones, 2006).

En segundo lugar, las «centralidades multiétnicas minoritarias» consisten en concentraciones de negociantes de diversas nacionalidades o etnias en barrios donde no suele predominar de forma clara un grupo coétnico residente. Es decir, de alguna manera existe una correspondencia entre la multietnicidad de los emprendedores y la multietnicidad de los residentes en el barrio. Li (1998 y 2007) ha caracterizado los ethnoburbs como suburbios multiétnicos. Li y sus colaboradores (2006) hablan de multiethnic settlements en Estados Unidos. Para Chacko y Cheung (2006), en ese país existen ciudades multiculturales con barrios con múltiples minorías. Estados Unidos ha entrado desde 1991 en una «nueva era dorada de barrios multiétnicos» (Denton y Massey, 1991, citados por Zelinsky y Lee, 1998). Por tanto, en este tipo de entornos multiétnicos es en donde se instalan las «centralidades multiétnicas minoritarias». En un barrio de Los Ángeles donde los residentes indios son minoritarios en relación a otras minorías de asiáticos y de latinoamericanos, la demarcación Little India no acaba de imponerse pese a la visible presencia de negocios indios (Chacko y Cheung, 2006). La mayor

2 La interacción entre grupos étnicos diferentes y de diferente estatus social degenera en ocasiones en malas relaciones, como en los disturbios de Los Ángeles de 1992. El libro de Lee (2002) estudia las relaciones entre emprendedores judíos o coreanos en barrios de afroamericanos en Estados Unidos. 
concentración de residentes bolivianos en Estados Unidos, en Virginia del Norte (área metropolitana de Washington D.C.), no ha obtenido tampoco el sello de Little Bolivia, puesto que en esos mismos barrios existen otros grupos étnicos (Price, 2006). En Queens y Brooklyn (Nueva York) se concentra la comunidad más numerosa de salvadoreños y de guatemaltecos en Estados Unidos; sin embargo, estos grupos no disponen de un «barrio propio» en esos distritos. Estos centroamericanos obtienen los productos de su tierra en comercios de otros «hispanos» (Miyares, 2007).

La Petite Asie de París consiste en otra «centralidad multiétnica minoritaria», si atendemos todavía hoy a los datos de Anne Raulin (2000) de 1990: la población asiática no constituía en esa fecha más que el 3.5\% del arrondissement o distrito, entre los inmigrantes, refugiados con frecuencia, procedentes de Camboya (3.201), Laos (873), Vietnam (740) y China-Taiwan (371). En cambio, el comercio era y es mayoritariamente asiático en una zona más reducida del barrio, en el Triangle de Choisy o Petite Asie. Los titulares de los comercios tienen unas procedencias similares a las de los propios residentes, es decir, nos hallamos ante una centralidad comercial de múltiples grupos étnicos de asiáticos en un barrio donde los asiáticos son una minoría multiétnica.

Dearborn, en los suburbios de Detroit (Michigan), es la ciudad norteamericana con mayor índice de población árabe, compuesta por palestinos, libaneses, iraquíes, yemeníes, etc. En la zona este de la ciudad los árabes constituyen hasta el 45\% de los distritos censales. Ahí es donde se localiza una «centralidad multiétnica minoritaria», con negocios de representantes de todas las nacionalidades árabes de la ciudad: el diario Arab American News e instituciones como siete mezquitas - una de ellas es de las mayores del país-y la cámara de comercio árabe-americana (Chacko, 2007).

El distrito de Ciutat Vella de Barcelona representa otro ejemplo de «centralidad multiétnica minoritaria». En este distrito, la inmigración es alta pero no mayoritaria, con alrededor de un 37\% de residentes extranjeros (en 2004) de múltiples nacionalidades: paquistaníes, marroquíes, dominicanos, filipinos, etc. Los emprendedores paquistaníes suponen aproximadamente en ese año un tercio del total de los negociantes étnicos. Les siguen los comerciantes indios (17\%), los emprendedores chinos (16\%), los marroquíes (9\%), los bengalíes y los filipinos (3\%), respectivamente, etc. (Serra, 2006).

El barrio Esquilino de Roma se ha transformado también en un espacio donde se concentran emprendedores de hasta 20 nacionalidades (Mundu, 2006). La rue del Faubourg Saint-Denis de París constituye un espacio de negocios multiétnicos, con la presencia de peluquerías, restaurantes, bares y tiendas de alimenta- 
ción, tanto de turcos como de indios y paquistaníes de diversos orígenes, tamiles de Sri Lanka, indo-mauricios, etc., que Vuddamalay (2007) denomina genéricamente Little India; se encuentran subespecializaciones como Little Jaffna, Little Punjab y el bazar o tchawke paquistaní. La presencia de los negocios turcos en ese mismo espacio ha llevado a Dinh (2002) a estudiar La petite Turquie. Dinh y Vuddamalay yuxtaponen y superponen por tanto las diferentes etnicidades de los espacios de negocios en un mismo entorno. De la misma manera, pienso que ese entorno se puede considerar globalmente o a una escala más pequeña como una «centralidad multiétnica minoritaria», en la que ningún grupo de emprendedores étnicos goza de predominio absoluto. Torres (2007) describe el barrio de inmigrantes de Russafa (Valencia) como «barrio multicultural», donde se se agrupan (en 2004) hasta 191 diferentes negocios étnicos en diversos ambientes: el chino (mayoristas textiles), el magrebí, el senegalés (mayoristas de ropa de cuero) y el latinoamericano. Para Torres, sólo en el caso de los magrebíes se da en Russafa una alta correspondencia entre lugar de residencia y concentración comercial.

A continuación, las «centralidades étnicas especializadas» suponen la concentración de negocios étnicos especializados. La especialización funcional es lo que más caracteriza a este tipo de centralidad. Lo más común es que las «centralidades étnicas especializadas» sean, por otro lado, «minoritarias». Las concentraciones de negocios de mayoristas textiles chinos en los barrios donde los chinos no son el principal grupo residente —Lavapiés en Madrid (Riesco, 2007); en la zona del Eixample, en torno a la calle Trafalgar, en Barcelona, y en el barrio de La Roquette (alrededor de las calles Sedaine y Popincourt), en París, así como el eje de tiendas de indios sindis que venden souvenirs en las Ramblas de Barcelona y el eje de restaurantes sudasiáticos de la Curry Mile en Manchester (Barret y McEvoy, 2006) - son ejemplos que encajan en la definición. En Londres, las minorías étnicas participan intensamente en la industria de la confección. Los diferentes grupos de emprendedores étnicos se dividen de manera espacial en barrios y por subsectores. Así, los grecochipriotas tienen su sector de vestido femenino en el norte de Londres, en los boroughs de Haringey e Islington sobre todo; los turcochipriotas, en el nordeste de Londres, con el sector de ropa de abrigo, en el borough de Hackney; los bengalíes trabajan las chaquetas y los abrigos de cuero en el este de Londres, en el borough de Tower Hamlets (Panyiatopoulos et alii, 2002).

Para Joaquín Beltrán (la cursiva es mía) el comercio textil al por mayor chino se caracteriza: 
por la pauta de su concentración en determinadas zonas; en Barcelona, en el límite entre los barrios del Eixample y de Ciutat Vella; en Madrid en el barrio de Lavapiés. La concentración de negocios dedicados a una actividad similar recuerda a los gremios de antaño y ha sido, y continúa siendo, una práctica habitual en la China de hoy en día: en las ciudades hay calles o barrios especializados en negocios concretos que todo el mundo conoce y adonde uno se dirige en caso de necesitar algo de lo que allí se ofrece. La concentración en este caso no parece afectar al éxito económico de las empresas, sino todo lo contrario. Las tiendas al por mayor se abastecen directamente de China o bien de los talleres que también se han establecido en España regidos por ellos mismos. En ocasiones las tiendas, igual que los talleres de confección, son empresas donde han invertido los propietarios de restaurantes ante la saturación creciente de la hostelería china y la acumulación de capital disponible que busca nuevas alternativas de inversión para aumentar sus beneficios. (Beltrán, 2000: 10).

\section{Los procesos de dispersión de emprendedores étnicos}

Las «zonas de emprendedores étnicos minoritarios» constituyen el cuarto tipo de espacio de negocios étnicos que se aparta de las centralidades étnicas descrito en este artículo. Las «zonas de emprendedores étnicos minoritarios» en el tejido urbano responden al fenómeno de las middlemen minorities (Bonacich, 1973), que Waldinger, McEvan y Aldrich (1990) describen como «doing business outside the ethnic community», con la particularidad de que tampoco suelen situarse en zonas donde predomina la población mayoritaria, sino en barrios poco atractivos para las franquicias comerciales y otros negocios a causa de la falta seguridad en las calles y el escaso poder adquisitivo de los vecinos. La dispersión permite, por tanto, disponer de una clientela no coétnica más amplia (Gober, 2000), puesto que la clientela coétnica puede ser insuficiente en ocasiones, como sucede en el Little Portugal de Toronto, cuya población portuguesa está en declive (Teixeira, 2006).

La diferencia básica entre las «centralidades étnicas minoritarias»y las «zonas de emprendedores étnicos minoritarios» es geográfica. En ambos casos el origen de los emprendedores es distinto del de los grupos étnicos residentes. En el primer caso, sin embargo, se produce una concentración de negocios en zonas o barrios delimitados; en el segundo se trata de una dispersión de negocios étnicos a escala metropolitana. 
Los grupos de emprendedores étnicos pueden utilizar las dos estrategias de concentración y de dispersión. Así, si un tercio de los emprendedores coreanos se concentra en la única Koreatown de Los Ángeles de los años ochenta del pasado siglo, también es cierto que los otros dos tercios de los negociantes coreanos se hallan dispersos en el $87 \%$ de las 10.000 zonas postales del condado de Los Ángeles (Waldinger et alii, 1990): «centralidad étnica minoritaria», por tanto, en Koreatown, compatible con una dispersión de negociantes étnicos a escala metropolitana donde la base de la clientela no es coreana (Reimer, 2007). En su conocido artículo, Bonacich (1973) ejemplifica su teoría precisamente con emprendedores coreanos. Para Reimer (2007), la teoría es limitada en el sentido de que Bonacich incluyó solo a hombres en su teoría sobre los middlemen minorities, como lo testimonian los 500 salones de manicura de coreanas dispersos en los cinco boroughs de Nueva York. Otro ejemplo de estrategia mixta lo constituye Phoenix, en Arizona, donde además de una pequeña Chinatown se dispersan numerosas tiendas de alimentación chinas repartidas en barrios donde los residentes mayoritarios son mexicanos ( $\mathrm{Li}, 2007)$.

Las «zonas de emprendedores étnicos minoritarios» suelen mostrar una especialización funcional, como las lavanderías chinas en Nueva York antes de 1965; los restaurantes chinos en París y Barcelona; los bazares chinos en Madrid; o bien, los comerciantes coreanos de comestibles de Nueva York, que monopolizaban este negocio en los años ochenta sobre todo en barrios menos favorecidos, mientras que ellos vivían en otros barrios mejores (Zhou, 1992). Waldinger et alii (1990) facilitan otros ejemplos de «emprendedores étnicos minoritarios» especializados de los años ochenta, como los restaurantes bengalíes sylheti en ciudades del Reino Unido y los bares, restaurantes y tiendas de venta de licores de los libaneses en Toledo (Ohio, EE.UU.).

En Nueva Orleans existe un conjunto de negocios más bien disperso en el centro urbano, en el West Bank, sobre todo de restaurantes controlados por vietnamitas que gozan de una demanda asiática amplia (Airriess, 2006). La dispersión permite, por tanto, disponer de una clientela más amplia (Light, 1998, citado por Gober, 2000), puesto que la clientela coétnica puede ser en ocasiones insuficiente.

Los restaurantes chinos son los clásicos ejemplos de «emprendedores étnicos minoritarios» especializados y dispersos, presentes en numerosas capitales europeas. La saturación de restaurantes chinos dispersos en el Reino Unido condujo a una emigración de restauradores chinos hacia la Europa continental septentrional, Alemania Occidental y Escandinavia hacia los años setenta. Esta ola migratoria especializada se traslada más adelante al sur de Europa, como des- 
taca Watson (1977, citado por Waldinger et alii, 1990). Beltrán (2000) desvela la estrategia espacial de los chinos en España a partir de los años setenta. Los restauradores chinos «acompañan» a los turistas de Europa Occidental al arco mediterráneo español y a Madrid y Barcelona, con la misma idea de la dispersión urbana. A medida que los destinos turísticos españoles se saturan, los chinos tienden a instalar sus restaurantes en localidades menores del interior, de en torno a los 10.000 habitantes. La dispersión se manifiesta por tanto a escala continental, estatal y regional como también a escala local. Beltrán censa más de 500 restaurantes chinos repartidos en Barcelona en el año 2000. Este autor subraya el alto nivel de competencia entre los restaurantes chinos, razón por la que persiguen la estrategia de la dispersión geográfica. Aun así:

En las capitales de provincia el número creciente de nuevos restaurantes que se abre llega un momento en que satura su oferta, es decir, son incapaces de mantener una clientela mínima que haga rentable y beneficiosa la empresa. La competencia entre restaurantes se exacerba con la bajada de los precios del menú, obligando a muchos de ellos a cerrar, incapaces de mantener unos márgenes de beneficios cada vez más precarios, o incluso inexistentes. El caso del municipio de Barcelona es el más espectacular: de varias docenas de restaurantes de comida china que había en 1990 se pasa en tres años a más de medio millar. Dos años después muchos han cerrado, incapaces de sobrevivir en una ciudad que no puede absorber tan rápido su oferta: de los 500 restaurantes de 1992, cerraron alrededor de 200 durante 1993-1995, aunque en la actualidad (2000), ya han vuelto a superar la cantidad de 1992 (Beltrán, 2000: 8).

Los paquistaníes, y en menor medida los bengalíes, están retomando los comercios de proximidad de alimentación en Barcelona. No se encuentran concentrados tan solo en el distrito de Ciutat Vella (dentro del cual, por cierto, buscan la dispersión), donde cuentan con su clientela coétnica, sino que se hallan además dispersos en barrios habitados en su mayor o en gran parte por autóctonos. En Barcelona, «anar al paki» significa en catalán ir al comercio de alimentación más cercano. Lo mismo sucede con los bazares chinos, donde se vende toda clase de productos domésticos baratos, tanto en Barcelona como en Madrid (Solé et alii, 2007). En España, ir al chino «significa» ir al bazar más próximo. «La dispersion assure à chaque secteur urbain un présence de proximité avec "son chinois", "son arabe", "son vietnamien", "son kabyle","son italien”» (Raulin, 2000: 18). En el francés coloquial también se habla de «l'arabe du coin» («el árabe de la esquina»).

La cartografía de los cerca de 900 restaurantes chinos de París en 1990 (Ma Mung, 2000) revela concentraciones en algunos distritos, en especial en el XIII, 
en la «centralidad multiétnica minoritaria» de la Petite Asie. Sin embargo, la mayoría de los restaurantes chinos se encuentran dispersos en todos los distritos. Concentración y dispersión urbana de la especialización funcional de la restauración china se manifiestan en París de manera compatible, simultánea.

En dos artículos (el presente y el de RIO, de junio de 2009) he expuesto cinco tipos de espacios de negocios étnicos: centralidades étnicas, centralidades multi/étnicas minoritarias, centralidades étnicas especializadas y emprendedores étnicos minoritarios. Cada tipo se caracteriza por una configuración o una combinación especifica de factores geográficos: concentración-dispersión, diversidadespecialización y la relación de los negocios étnicos respecto a la mayoría de los residentes inmigrantes.

Las tablas 1 y 2 muestran respectivamente algunos ejemplos de los cinco tipos de espacios de negocios étnicos, así como los procesos geográficos que se manifiestan de manera más palpable en esos tipos de espacios.

Tabla 1. Tipos de espacios de negocios étnicos y algunos ejemplos

\begin{tabular}{|l|l|}
\hline \multicolumn{1}{|c|}{$\begin{array}{c}\text { Tipologías de espacios } \\
\text { de negocios étnicos }\end{array}$} & \multicolumn{1}{c|}{ Algunos ejemplos } \\
\hline Centralidades étnicas & $\begin{array}{l}\text { Chinatowns de Nueva York, Sydney y San Francisco/ } \\
\text { Kreuzberg de Berlín/ «Barrios» de Phoenix/Little } \\
\text { Portugal de Toronto/Banglatown de Londres }\end{array}$ \\
\hline $\begin{array}{l}\text { Centralidades étnicas } \\
\text { minoritarias }\end{array}$ & $\begin{array}{l}\text { Little Italy y Asiatown, en Sydney/Los Ángeles } \\
\text { Koreatown/Faubourg de Montmartre y Quartier Juif, } \\
\text { en París }\end{array}$ \\
\hline $\begin{array}{l}\text { Centralidades multiétnicas } \\
\text { minoritarias }\end{array}$ & $\begin{array}{l}\text { Ciutat Vella de Barcelona/Esquilino de Roma/Rue } \\
\text { Faubourg Saint-Denis y Petite Asie, en París }\end{array}$ \\
\hline $\begin{array}{l}\text { Centralidades étnicas } \\
\text { especializadas }\end{array}$ & $\begin{array}{l}\text { mayoristas textiles chinos; Lavapiés, Madrid; } \\
\text { La Roquette, París; Trafalgar, Barcelona/Souvenirs de } \\
\text { sindis en Las Ramblas, Barcelona }\end{array}$ \\
\hline $\begin{array}{l}\text { Zonas de emprendedores } \\
\text { étnicos minoritarios }\end{array}$ & $\begin{array}{l}\text { coreanos en el condado de Los Ángeles/restaurantes } \\
\text { chinos en París y Barcelona/bazares chinos en Madrid }\end{array}$ \\
\hline
\end{tabular}


Tabla 2. Tipos de espacios de negocios étnicos y procesos geográficos correspondientes

\begin{tabular}{|l|c|c|c|}
\hline $\begin{array}{c}\text { Tipologías de espacios } \\
\text { de negocios étnicos }\end{array}$ & $\begin{array}{c}\text { Tipo de distribución } \\
\text { geográfica de negocios } \\
\text { coétnicos o no coétnicos }\end{array}$ & $\begin{array}{c}\text { Tipo de } \\
\text { actividades de los } \\
\text { negocios étnicos }\end{array}$ & $\begin{array}{c}\text { Presencia } \\
\text { de residentes } \\
\text { coétnicos en la zona }\end{array}$ \\
\hline Centralidades étnicas & $\begin{array}{c}\text { Concentración de } \\
\text { negocios coétnicos }\end{array}$ & Diversificación & Mayoría \\
\hline $\begin{array}{l}\text { Centralidades étnicas } \\
\text { minoritarias }\end{array}$ & $\begin{array}{c}\text { Concentración de } \\
\text { negocios coétnicos }\end{array}$ & Diversificación & Minoría \\
\hline $\begin{array}{l}\text { Centralidades } \\
\text { multiétnicas } \\
\text { minoritarias }\end{array}$ & $\begin{array}{c}\text { Concentración de } \\
\text { negocios coétnicos y no } \\
\text { coétnicos }\end{array}$ & Diversificación & Minoría \\
\hline $\begin{array}{l}\text { Centralidades étnicas } \\
\text { especializadas }\end{array}$ & $\begin{array}{c}\text { Concentración de } \\
\text { negocios coétnicos }\end{array}$ & Especialización & Minoría \\
\hline $\begin{array}{l}\text { Zonas de } \\
\text { emprendedores } \\
\text { étnicos minoritarios }\end{array}$ & $\begin{array}{c}\text { Dispersión de negocios } \\
\text { coétnicos }\end{array}$ & Especialización & Minoría \\
\hline
\end{tabular}

Las fronteras entre unos espacios de negocios étnicos y otros pueden ser, en ocasiones, borrosas. Puede ocurrir, además, que unas tipologías evolucionen hacia otras y hasta pueden lógicamente crear «mutaciones» no previstas. En cualquier caso, las tipologías que he presentado aquí no pretenden reducir la realidad a algunos estados hipotéticamente «puros» y estables.

\section{Conclusiones}

En los dos artículos he tratado de mostrar que concentración y dispersión, especialización y diversidad, son procesos de carácter eminentemente geográfico que intervienen en los espacios de negocios étnicos. He intentado caracterizar cinco tipologías de espacios de negocios étnicos: centralidades étnicas, centralidades étnicas minoritarias, centralidades multiétnicas minoritarias, centralidades étnicas especializadas y zonas de emprendedores étnicos minoritarios. En los cuatro primeros tipos, en las «centralidades étnicas», predominan sobre todo los procesos de concentración de los negocios étnicos. En cambio, los «emprendedores étnicos minoritarios» presentan siempre una distribución geográfica dispersa.

En las «centralidades étnicas minoritarias» se concentran negocios de un mismo grupo étnico en un área residencial dominada por otro grupo no coétnico, como Koreatown en Los Ángeles. En las «centralidades multiétnicas minoritarias», como la de Ciutat Vella en Barcelona, no predominan los negocios de un 
mismo grupo étnico sino de múltiples grupos, en un barrio donde los residentes de los grupos étnicos correspondientes también constituyen múltiples minorías. La diversidad de los negocios caracteriza a estos tres tipos de centralidades étnicas. En las «centralidades étnicas especializadas» se produce una alta densidad de negocios normalmente de un mismo grupo étnico, destinados a un mismo sector económico, como los mayoristas chinos en La Roquette, en París. La especialización funcional distingue por tanto a esta centralidad étnica de las otras.

En el caso de los espacios de «emprendedores étnicos minoritarios» predomina el fenómeno de la dispersión metropolitana, al contrario que en las cuatro centralidades étnicas. Los «emprendedores étnicos minoritarios» están dispersos en una ciudad o en una zona amplia donde los residentes forman parte de otro grupo étnico, como los restaurantes chinos dispersos en tantas ciudades europeas.

En cuanto a posibles líneas de investigación, se podría realizar un estudio comparativo de los espacios de negocios étnicos de las principales capitales europeas con objeto de aplicar de manera empírica las tipologías y procesos que he sugerido. Una investigación de ese estilo puede implicar algunos desafíos o dificultades, como la correcta utilización de escalas geográficas apropiadas para analizar espacios de negocios étnicos en áreas metropolitanas de extensiones y densidades diferentes. La utilización de grupos étnicos agregados (como la categoría Hispanics o latinos del censo de los EE.UU.) o desagregados, tanto residentes como emprendedores, también constituye un aspecto a tener en cuenta a la hora de clasificar un espacio de negocios étnicos como una centralidad étnica o multiétnica.

\section{Bibliografía}

Airriess, C. (2007). «Conflict Migrants From Mainland Southeast Asia». En Miyares, I. y Airriess, C.A., Contemporary Ethnic Geographies in America. Rowman E Littlefield, pp. 291-312.

Airriess, C. (2006). «Scaling Central Place of an Ethnic-Vietnamese Commercial Enclave in New Orleans, Louisiana». En Kaplan, D. H. y Li, W. Landscapes of the Ethnic Economy. Plymouth (Reino Unido): Rowman \& Littlefield, pp. 17-33.

Barret, G. A. y McEvoy, D. (2006). "The Evolution of Manchester's Curry Mile: From Suburban Shopping Street to Ethnic Destination». En Kaplan, D. H. y LI, W., Landscapes of the Ethnic Economy. Plymouth: United Kingdom: Rowman \& Littlefield, pp. 193-208. 
Beltrán Antolín, J. (2000). «Expansión geográfica y diversificación económica. Pautas y estrategias del asentamiento chino en España». Actas del II Congreso sobre la Inmigración en España. España y las migraciones internacionales en el cambio de siglo. Madrid: Instituto Universitario de Estudios sobre Migraciones de la Universidad Pontificia Comillas/Instituto Universitario Ortega y Gasset. Disponible en: <sirio.ua.es/documentos/pdf/flujos_migratorios/expansion\%20geaogrfaica\%20y\%20diversificacion.pdf> [Consulta: 3-XII-2007].

Bonacich, E. (1973). «A theory of middlemen minorities»+ American Sociological Review, 38: 585-594.

Boswell, T. D. y Jones, T-A. (2006). «The Distribution and Socioeconomic Status of West Indians Living in the United States». En Frazier, J. W. y Tettey-Fio, E. L. (ed.). Race, Ethnicity, and Place in a Changing America. Binghamton (NY, EE.UU.)ः Global Academic Publishing, págs. 155-180.

Brody, J. (1995). Rue des Rosiers: une manière d'être juif. Autrement. (Colección Français d'ailleurs, peuple d'ici, serie Monde, número 82).

Chacko, E. (2007). «Immigrants from the Muslim World: Lebanese and Iranians». En Miyares, I. y Airriess, C. A. Contemporary Ethnic Geographies in America. Plymouth, United Kingdom: Rowman \& Littlefield, pp. 313-330.

Chacko, E. y Cheung, I. (2006). «The Formation of a Contemporary Ethnic Enclave: The Case of «Little Ethiopia» in Los Angeles». En Frazier, J. W. y Tettey-Fio, E. L. (ed.). Race, Ethnicity, and Place in a Changing America. Binghamton (NY, EE.UU.)ः Global Academic Publishing, pp. 131-139.

Collins, J. (2006). «Ethnic Diversity and the Ethnic Economy in Cosmopolitan Sydney». En Kaplan, D. H. y LI, W. Landscapes of the Ethnic Economy. Plymouth, United Kingdom: Rowman \& Littlefield, pp. 135-148.

Denton, N.A. y Massey, D.S. (1991). «Patterns of neighborhood transition in a multiethnic world». Demography, 28:41-63.

Dinh, M. B. (2002). La petite Turquie, transposition de villages en situation migratoire. DEA de geografia urbana de la Université de Paris X-Nanterre.

DJERgaÏAN, S. (2007). «Un territoire de l'identité arménienne. Le 9e arrondissement de París». Homes et Migrations, 1265: 54-69.

Gober, P. (2000). «Immigration and North American Cities». Urban Geography, $21(1): 83-90$.

LI, W. (2007)+ «Chinese Americans: Community Formation in Time and Space». En Miyares, I. y Airriess, C.A., Contemporary Ethnic Geographies in America. Plymouth, United Kingdom: Rowman \& Littlefield, pp. 213-232.

LI, W.(1998). "Anatomy of a new ethnic settlement: the Chinese Ethnoburb in Los Angeles». Urban Studies, 35 (1): 79-502. 
Li, W.; Dymski, G.; Chee, M.; W.L.; Ahn, Hy-H.; Aldana, C., y Zhou, Y. (2006). «How Ethnic Banks Matter: Banking and Community/Economic Development in Los Angeles». En Kaplan, D.H. y Li, W. Landscapes of the Ethnic Economy. Plymouth, United Kingdom: Rowman \& Littlefield, págs. 113-134.

Light, I. y Bonacich, E. (1988). Immigrant Entrepreneurs: Koreans in Los Angeles, 1965-82. Berkeley: University of California Press.

Ma Mung, E. (2000). La diaspora chinoise: géographie d’une migration. París: GéOphrys, Ophrys.

Miyares, I. (2007). «Central Americans: Legal Status ands Settledness». En Miyares, I. y Airriess, C.A. Contemporary Ethnic Geographies in America. Plymouth, United Kingdom: Rowman \& Littlefield, pp. 175-190.

Mundu, P. P. (2006), «The New Romans; Ethnic Econmic Activities in Rome»* En Kaplan, D. H. y Li, W., Landscapes of the Ethnic Economy. Plymouth, United Kingdom: Rowman \& Littlefield, pp. 165-176.

Panayiotopoulos (aka Mike Pany), P. y Dreef, M. (2002). «London: Economic Differentiation and Policy-Making». En Ratr, J. Unravelling the Rag Trade: Immigrant Entrepreneurship in Seven World Cities. Oxford: Berg Publishers, pp. 49-72.

Raulin, A. (2000). L'ethnique est quotidien: diasporas, marchés et cultures métropolitaines. Paris: Connaissance des hommes, L'Harmattan.

Reimer, D. G. (2007). «Korean Culture and Entrepreneurship». En Miyares, I. y Airriess, C.A. (2007a). Contemporary Ethnic Geographies in America. Plymouth, United Kingdom: Rowman \& Littlefield, pp. 233-250.

Rekers, A. y VAn Kempen, R. (2000). «Location Matters: Ethnic Entrepreneurs and the Spatial Context». En Rath, J. (ed.). Immigrant business: the economic, political, and social environment. New York: Palgrave MacMillan, pp. 54-69.

Riesco Sanz, A. (2007). «Dimensiones y características de la empresarialidad inmigrante en Lavapiés (Madrid)». IV Congreso de la Inmigración. Valencia.

Serra del Pozo, P. (2006). El comercio étnico en el distrito de Ciutat Vella en Barcelona. Barcelona: Fundació La Caixa.

Teixeira, C. (2006). «Residential Segregation and Ethnic Economies in a Multicultural City: The Little Portugal of Toronto». En Kaplan, D. H. y Li, W. Landscapes of the Ethnic Economy. Plymouth, United Kingdom: Rowman \& Littlefield, pp. 49-65.

Torres Pérez, F. (2007). Nous veïs a la ciutat. Els immigrants a València i Russafa. Valencia: Universitat de València. 
Vuddamalay, V. (2007). «Inner-city Ethnic Enclaves and Irregular Immigrant Networks as New Social Spaces in Global Cities: A Case-study of the "Little India" of París». En Berggren, E.; Likić-Brborić, T., y Trimikliniotis, G. Irregular Migration, Informal Labour and Community: a Challege for Europe. Maastrich: Shaker Publishing.

Waldinger, R.; McEvoy, D., y Aldrich, H. (1990), «Spatial dimensions of oportunity structures», en Waldinger, R.; Aldrich, H., y Ward, R. Ethnic entrepreneurs: Immigrant business in industrial societies. Newbury Park, CA: Sage Publications.

Watson, J. (1977). Between Two Cultures: Migrants and Minorities in Britain. London: Blackwell.

Zelinsky, W. y Lee, B.A. (1998). «Heterolocalism: An Alternative Model of the Siociospatial Behaviour of Immigrant Ethnic Communities». International Journal of Population Geography, 4: 281-298.

Zhou, M. (2004). «Revisiting Ethnic Entrepreneurship: Convergencies, Controversies, and Conceptual Advancements». International Migration Review, 38: 1040-1074.

- (1992). «New York's Chinatown: The Socioeconomic Potential of an Urban Enclave». Philadelphia: Temple University Press. 